\title{
The electrochemical determination of formaldehyde in aqueous media using nickel modified electrodes.
}

Dhruv Trivedi, a John Crosse, ${ }^{b}$ Jonathon Tanti, ${ }^{a}$ Alise J Cass, ${ }^{a}$ Kathryn E. Toghilla ${ }^{*}$

a Department of Chemistry, Lancaster University, Lancaster, UK

${ }^{b}$ Lancaster Environmental Centre, Lancaster University, Lancaster, UK

\begin{abstract}
Glassy carbon (GC) electrodes were modified with nickel metal via a simple deposition procedure, followed by enrichment of the nickel in a potassium hydroxide solution to deliver the catalytic nickel hydroxide species $\left(\mathrm{Ni}(\mathrm{OH})_{2}\right)$. In solutions of $1 \mathrm{M} \mathrm{KOH}$, the nickel modified GC electrode (Ni-GC) contained a reproducible detection limit of the order of 1.1 $\times 10^{-5} \mathrm{M}$ for formaldehyde additions. This is comparable and, in many cases, surpasses, platinum group metal modified electrodes. The potentiometric analytical method also allowed for the accurate determination of "unknown" formaldehyde concentrations, over a linear range of $1 \times 10^{-5}$ to $1 \times 10^{-3} \mathrm{M}$ and a sensitivity of $22.7+/-3.8 \mu \mathrm{A} / \mathrm{mM}$. Furthermore, the $\mathrm{Ni}-\mathrm{GC}$ electrode showed negligible response to formate and methanol, even when they were present in concentrations 10 times greater than the formaldehyde. The electrochemical performance was compared to a simple colorimetric approach to formaldehyde determination, wherein a detection limit of $6 \times 10^{-6} \mathrm{M}$ was obtained.
\end{abstract}

\section{Keywords:}

Nickel catalyst; electrocatalysis; formaldehyde; non-enzymatic; $\mathrm{CO}_{2}$ reduction; Hantzsch

\section{Introduction}

Formaldehyde is used worldwide in large quantities as a raw material in the production of chemicals and plastics manufacturing, as well as in various household products.[1] This extensive industrial and domestic usage means that it is present in workplace air spaces, industrial waste materials, and also a prevalent contaminant in ground water due to 
dumpsite leaching.[1-3] Formaldehyde is also a potential product in the electrocatalytic conversion of $\mathrm{CO}_{2}$ to produce useful hydrocarbon synthetic fuels, such as methanol and methane, directly from renewable electricity.[4] The complex multi-proton, multi-electron reduction of $\mathrm{CO}_{2}$ yields an array of small organic products; the first three water-soluble products being formate, formaldehyde and methanol.[4] A simple, selective and effective means of identifying and quantifying these small organic molecules, in a typically complex reaction medium, is a fundamental issue [5] - most notably for formaldehyde [6-8]. Developing methods to selectively, accurately and rapidly identify and quantify these products would therefore be of great advantage to $\mathrm{CO}_{2}$ reduction research, not to mention a valuable analytical development in wastewater treatment and analysis. [9, 10]

Varieties of analytical strategies exist to determine formaldehyde, with emphasis typically being on the determination of gas phase formaldehyde. Of the liquid phase determinations, the most notable technique is the derivatization of the carbonyl compound with 2,4 - dinitrophenylhydrazine (2,4-DNPH). This compound is commonly known to produce Brady's reagent for the qualitative determination of aldehydes. It is also the reagent stipulated by the U.S. Environmental Protection Agency [11] to be used quantitatively in conjunction with high performance liquid chromatography (HPLC). The analytical method requires a lengthy derivatization process and the use of reversed-phase (RP-HPLC).[4, 11, 12] The approach is the analytical state-of-the-art, and obtains detection limits of $10^{-10} \mathrm{M}$ in an optimized system [13]. However, recent safety concerns have rendered this technique a somewhat problematic approach to formaldehyde determination, due to the flammable and explosive properties of the 2,4-DNPH when allowed to dry out in poor storage conditions. Consequently, purchasing the solid reagent is now difficult.

Similar to the 2,4-DNPH derivatization method, a lesser reported derivatization approach which is solely spectrophotometric is the adapted Hantzsch reagent method reported by Nash [14]. This approach uses the reaction between formaldehyde and acetylacetone, acetic acid and ammonium acetate to form diacetyldihydrolutidine (DDL), a yellow derivative of the formaldehyde with a high extinction coefficient.

Electrochemical approaches would better suit quantification of the complex electrolyte expected of $\mathrm{CO}_{2}$ reduction samples. Such samples are unsuitable for chromatographic machinery, and sample preparation would potentially cause sample loss. A small body of research exists in the field of electrochemical formaldehyde determination, with approaches typically using platinum [15, 16], palladium [17-21] or gold [22] 
electrocatalysts. Typically these studies are conducted in sulfuric acid or sodium hydroxide solution, and use various nanoarchitectures and complex fabrication methods in their design. Detection limits are typically of the order of $10^{-5} \mathrm{M}$, though some palladium electrodes have been reported to determine formaldehyde concentrations as low as $10^{-11}$ M. [16]

Nickel modified electrodes in alkaline solution are well-known catalysts towards small organic molecules. The $\mathrm{Ni}(\mathrm{III})$ species in the oxidised $\mathrm{NiOOH}$ readily reacts with organic compounds, oxidizing the organic analyte and reforming the $\mathrm{Ni}(\mathrm{OH})_{2}$ species [2326]. Despite the wealth of literature utilizing the $\mathrm{Ni}(\mathrm{OH})_{2}$ redox catalyst in alcohol and glucose oxidation,[27-29] very few researchers have considered the electrochemical oxidation of formaldehyde. Of the few that have [30-32] their focus has been on large concentrations in fuel cell assessment, as opposed to being used for formaldehyde detection.[33] As one would expect, the nickel catalyst strongly responds to the presence of formaldehyde. This is to be expected, as the formaldehyde forms a gem diol in water, and such polyol species are highly responsive to the nickel catalyst. [26]

Here in we report a simple, low cost nickel modified glassy carbon electrode and its application to formaldehyde determination. For parity, the analysis of formaldehyde by a spectrophotometric method is also discussed, and the merits and disadvantages of the two techniques considered.

\section{Experimental}

\subsection{Reagents and Equipment:}

Ammonium acetate, acetic acid, sodium acetate, and acetylacetone were reagent grade from Sigma Aldrich (UK) and used as received. $\mathrm{Ni}\left(\mathrm{NO}_{3}\right)_{2}$ was purchased from Sigma Aldrich (UK) and $\mathrm{KOH}$ was purchased from Fisher Scientific. All solutions were prepared using Milli- $\mathrm{Q}^{\circledR}$ ultrapure water of resistivity $18.2 \mathrm{M} \Omega \mathrm{cm}$.

The formaldehyde additions were made using a standardized formaldehyde stock solution. Formaldehyde $(\mathrm{HCOH})$ was purchased from ACROS Organics (37 wt \%, stabilized with $5-15 \%$ methanol). A $0.05 \mathrm{M}$ stock solution was prepared for use in electroanalytical experiments using Milli- $Q^{\circledR}$ ultrapure water. The stock was standardized following the US EPA Method 554 [34]. Anhydrous sodium sulfite (98\%) and hydrochloric acid (37\%) were purchased from Sigma Aldrich (UK) and used as received. 
To determine formaldehyde concentrations in a real water sample, pond water was obtained from the university campus, filtered to $0.45 \mu \mathrm{m}$, and spiked with a volume of formaldehyde stock solution.

Prior to use, all glassware was soaked for 8 hours in $3 \mathrm{M}$ hydrochloric acid followed by 3 rinses with Milli- $Q^{\circledR}$ ultrapure water $(18.2 \mathrm{M} \Omega$; organic carbon $<2 \mathrm{ppm})$. All electrochemical measurements were made using an Ivium EmSTAT 3+ (Alvatek, UK) in conjunction with the software PSTrace. The working electrodes were glassy carbon ( $3 \mathrm{~mm}$ $\varnothing)$, counter electrode was a platinum wire $(\mathrm{CH}$ Instruments, both purchased from IJ Cambria Scientific Ltd, UK), and a $\mathrm{Ag} / \mathrm{AgCl}$ reference electrode (BASi, Alvatek, UK). UV-vis experiments were made using a Jenway 7315 Spectrophotometer.

\subsection{Electrochemical method}

The nickel modified glassy carbon (Ni-GC) electrode was fabricated via the electrodeposition of nickel at $-1.3 \mathrm{~V}$ vs. $\mathrm{Ag} / \mathrm{AgCl}$ from a $1 \mathrm{mM} \mathrm{Ni}\left(\mathrm{NO}_{3}\right)_{2}$ in $0.1 \mathrm{M}$ acetate buffer deposition solution. The freshly polished GC electrode was held at potential from 30 or $600 \mathrm{~s}$ under constant stirring and under a nitrogen atmosphere. At higher deposition times a thin metal film was visible across the GC surface.

Following deposition, the Ni-GC electrode was removed from the deposition solution, washed with Milli- $Q^{\circledR}$ ultrapure water, and then placed in a $1 \mathrm{M} \mathrm{KOH}$ solution for conditioning. The electrode was cycled between 0.15 and $0.55 \mathrm{~V}$ in the alkaline medium ca. 200 times at a scan rate of $100 \mathrm{mVs}^{-1}$. This allowed for the crystalline phases of the $\mathrm{Ni}(\mathrm{OH})_{2}$ to settle into the aged beta phase [23-26]

At a holding potential of ca. $0.46 \mathrm{~V}$ vs. $\mathrm{Ag} / \mathrm{AgCl}$, determined from cyclic voltammograms in the presence of formaldehyde, a potentiometric calibration plot was obtained over various linear ranges. In a standard three-electrode set-up, under constant, fast stirring, additions of formaldehyde were made to the $1 \mathrm{M} \mathrm{KOH}$ solution at intervals of 20 to 30 seconds. A calibration plot was then produced based on the average current of the time interval of each addition. The method was then repeated with formaldehyde-spiked pond water.

\subsection{Spectrophotometric method}

2.3.1. By UV Detection 
A six-point calibration was made diluting the 35\% formaldehyde stock with Milli-Q ${ }^{\circledR}$ ultrapure water. Aqueous formaldehyde calibration standards were reacted with equal amounts of Hantzsch reagent containing 15\% w/v ammonium acetate, $3 \% \mathrm{v} / \mathrm{v}$ acetic acid and $2 \% \mathrm{v} / \mathrm{v}$ acetyl acetone, heated for 30 minutes in a water bath at $40^{\circ} \mathrm{C}$ and allowed to cool to room temperature $\left(20^{\circ} \mathrm{C}\right)$ for 30 minutes. The resultant yellow solution, $D D L$, (3, 5-diacetyldihydrolutidine) was then analysed by spectrophotometry at a wavelength of $412 \mathrm{~nm}$.

\subsubsection{Coupled with HPLC}

Following reaction of the formaldehyde with the Hantzsch reagent, the DDL solution was transferred to a GC vial and complementary DDL determination was performed using HPLC. Samples were run on an Agilent 1220 HPLC (Hanover, Germany) fitted with an Agilent Poroshell 120 EC-C18 column (3.0 x 50 mm; $2.7 \mu \mathrm{m}$ particle size), using a variable wavelength detector monitoring absorbance at $412 \mathrm{~nm}$. $20 \mu \mathrm{L}$ of sample was injected. The solvents were acetonitrile $(B)$ and water $(A)$.

\section{Results and discussion}

\subsection{Electrochemical determination of formaldehyde}

\subsubsection{Nickel deposition and conditioning}

In accordance to the method outlined in the experimental section, glassy carbon electrodes were modified with nickel films to produce a Ni-GC electrode. Optimisation of the deposition procedure found $-1.3 \mathrm{~V}$ vs $\mathrm{Ag} / \mathrm{AgCl}$ to be the best deposition potential, with a deposition time of $60 \mathrm{~s}$. It was necessary to avoid the formation of bubbles (caused by the reduction of water) on the electrode surface to ensure a smooth deposition of nickel. Deposition for 300 $s$ or more generated a relatively thick metal film on the electrode surface. Although the quantity of nickel was evidentially greater, the Ni-GC electrodes were found to be less durable to repetitive testing with more material.

Figure 1 shows a typical cyclic voltammogram for the Ni-GC electrode in $\mathrm{KOH}$ solution on the first cycle after deposition, and the final, $200^{\text {th }}$ cycle. The enrichment step is required with Ni-modified electrodes to ensure that the $\mathrm{Ni}(\mathrm{OH})_{2}$ layer is formed and present in the stable $\beta$-crystalline structure [23-26]. The anodic shift and growth of the broad $\mathrm{Ni}(\mathrm{OH})_{2}$ peak as the nickel oxidises to $\mathrm{NiOOH}$ is evident in Figure 1. Approximately 200 
cycles over the potential window ensured that the redox couple was stable and unchanging for the subsequent formaldehyde additions.

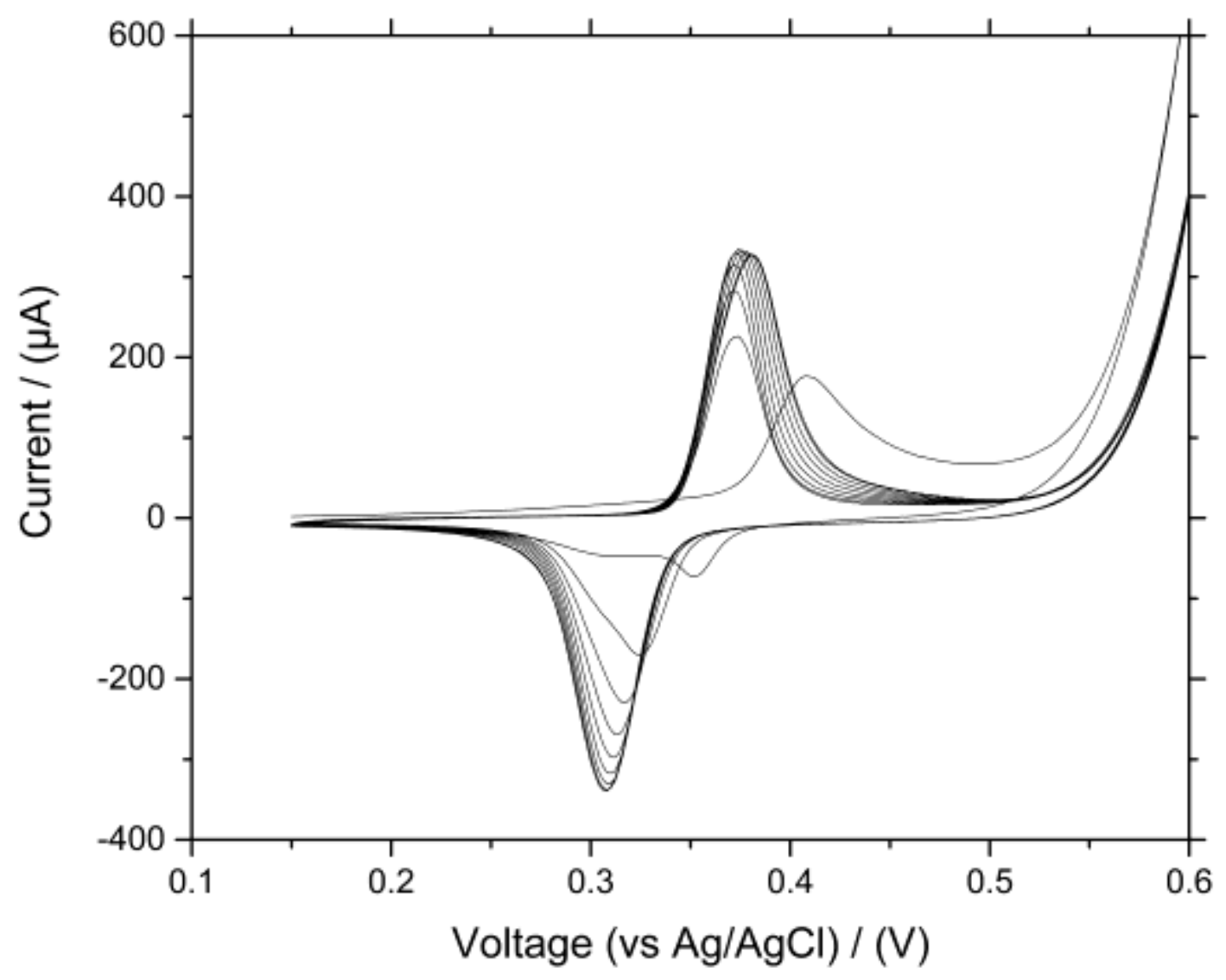

Figure 1: Enrichment of a nickel modified glassy carbon electrode for 250 scans in $1 \mathrm{M}$ $\mathrm{KOH}$ following $60 \mathrm{~s}$ deposition of nickel. Scan Rate: $0.2 \mathrm{~V} / \mathrm{s}$. Every 30th scan shown.

\subsubsection{Calibration plots and detection limits}

Figure 2 shows an overlay of cyclic voltammograms (CVs) taken of the Ni-GC electrode with increasing additions of $0.5 \mathrm{mM}$ formaldehyde. The electrocatalytic response to formaldehyde additions is evident in the forward scan, with the peak potential shown to shift positively with each large addition. Due to this shifting of the peak, and following procedures already noted in the literature [23-26], a target potential for potentiometric analysis of $0.46 \mathrm{~V}$ was chosen. 


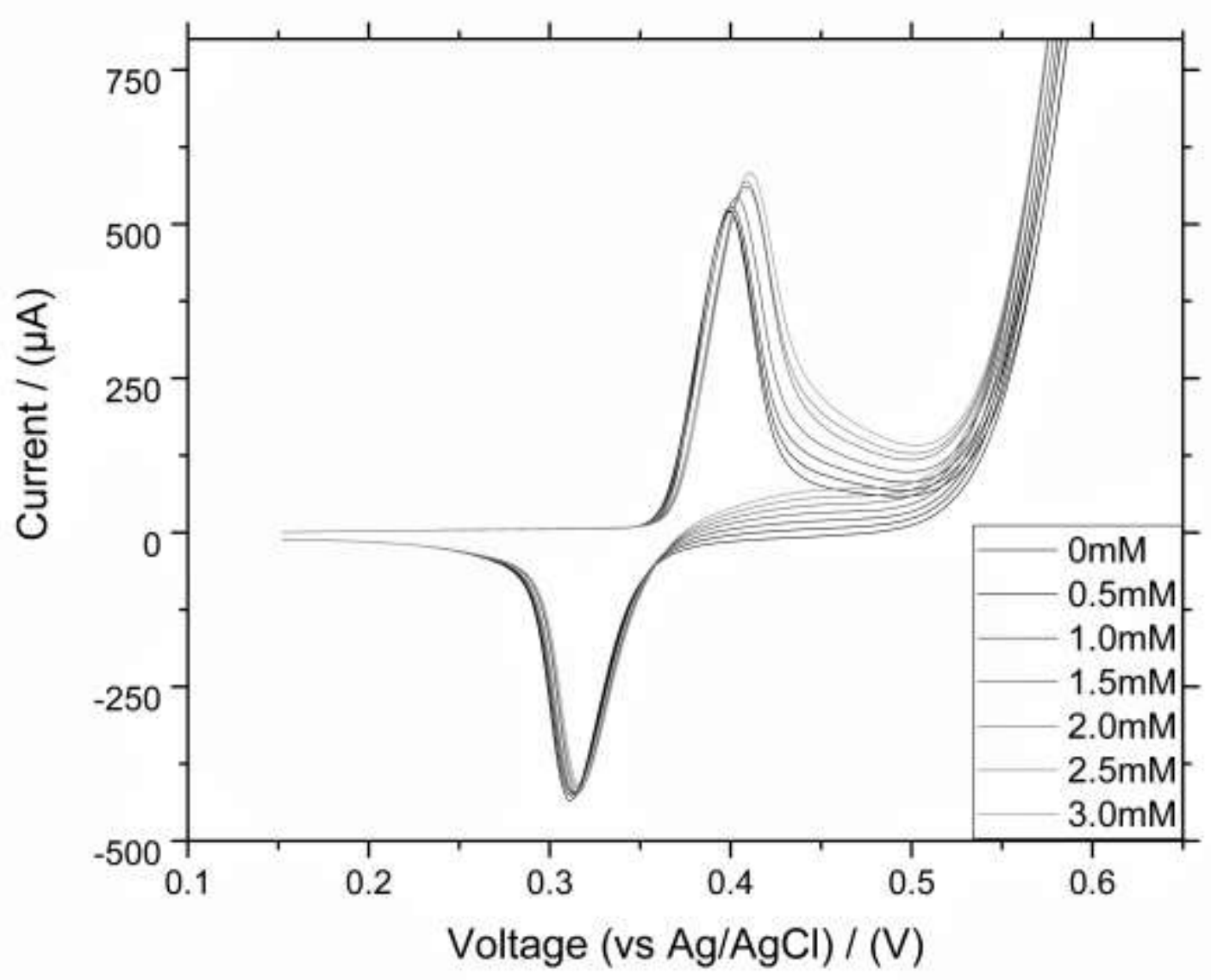

Figure 2: Six consecutive additions of $0.5 \mathrm{mMHCHO}$ to a $10 \mathrm{~mL} 1 \mathrm{M} \mathrm{KOH}$ solution. Scan Rate: $0.2 \mathrm{~V} / \mathrm{s}$; Electrode: Nickle modified GCE - $60 \mathrm{~s}$

Calibration plots for formaldehyde were obtained using a potentiometric staircase method under constant stirring. Figure 3 shows two typical plots over a linear range of $0.1-$ $1 \mathrm{mM}$ (Figure 3a) and $0.01-0.1 \mathrm{mM}$ (Figure 3b). The main figure shows the raw data acquired over the time interval, in which the noise on the current response may be attributed to the action of the stirring bar. In-set of each figure are the point calibration plots obtained by averaging the current response over each addition time interval. Both plots give a highly linear response of $R^{2}=0.999$, with a sensitivity of $19 u A m^{-1}$ for the $0.1 \mathrm{M}$ additions, and $24 \mathrm{uA} / \mathrm{mM}$ for the $0.01 \mathrm{mM}$ additions. The detection limit for both concentration ranges was $1 \times 10^{-5} \mathrm{M}$ calculated using $3 \sigma$ [35].

The detection limit and sensitivity shown here and obtained in many other experiments were of similar magnitude, giving a sensitivity of $22.8 \pm 3.8 \mu \mathrm{A} \mathrm{mM}^{-1}$ across both concentration ranges and an LOD range of $1.1-1.6 \times 10^{-5} \mathrm{M}$. The electrode was found 
to be especially durable for repeated use when the lower deposition time of $60 \mathrm{~s}$ had been employed. A simple 20 cycle reconditioning step in $\mathrm{KOH}$ solution reestablished the $\mathrm{Ni}(\mathrm{OH})_{2}$ layers, and a current response equivalent to the first potentiometric study could be obtained.

(a)

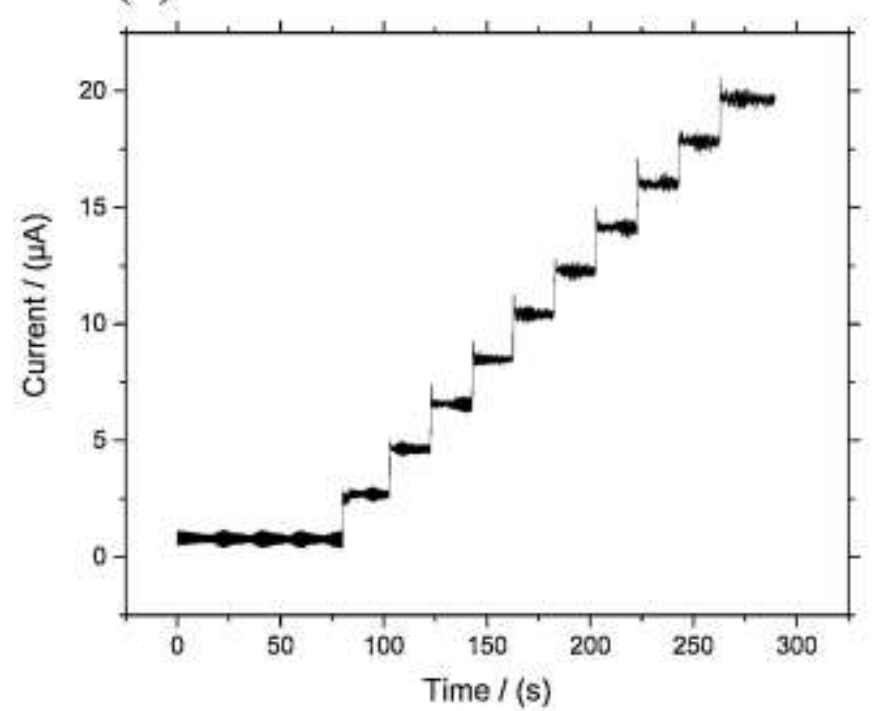

(b)

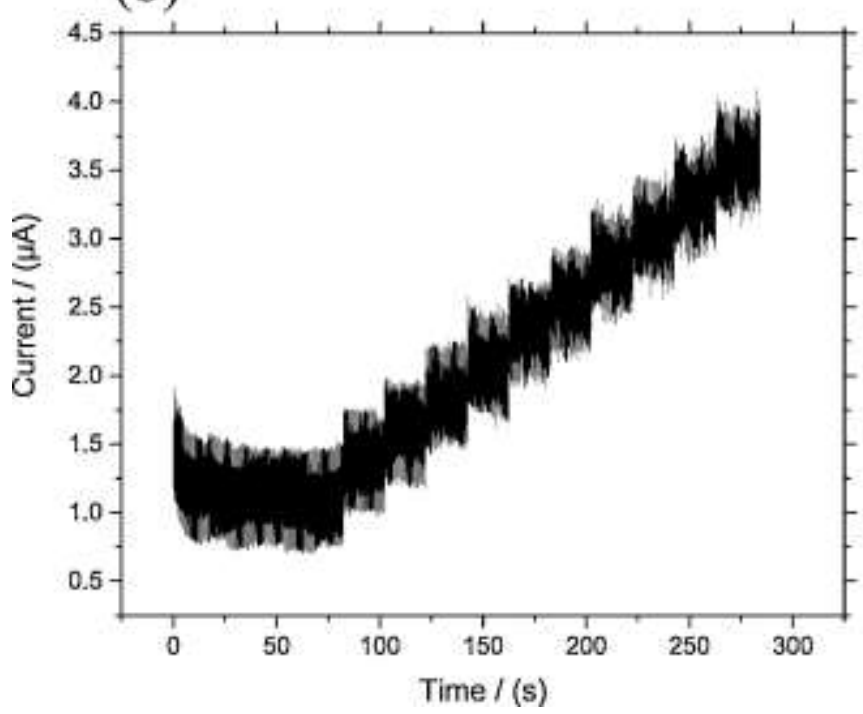

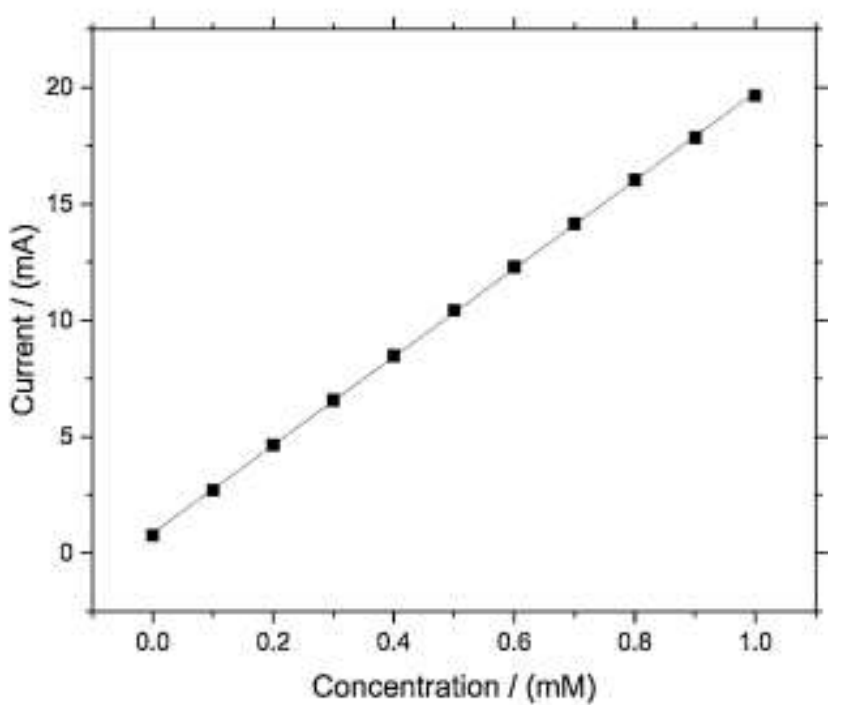

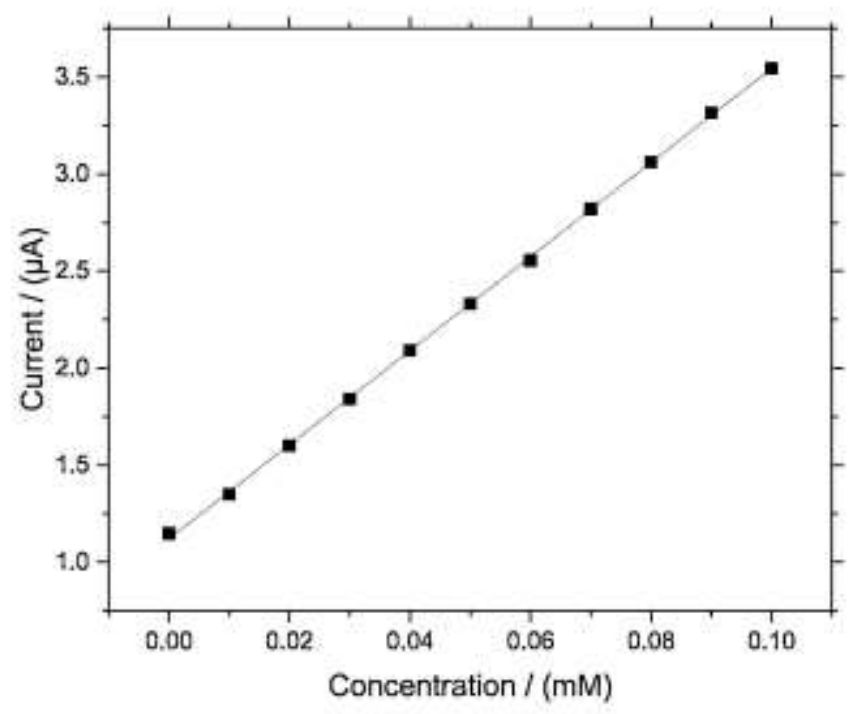

Figure 3: Standard additions of formaldehyde under chronoamperometric conditions, using the Ni-GC electrode under constant, fast stirring. a) Additions of $0.1 \mathrm{mM}$ formaldehyde with the calibration plot in-set. b) Additions of $0.01 \mathrm{mM}$ formaldehyde with corresponding calibration plot in-set 


\subsubsection{Determination of unknown concentrations}

As an extension of the calibration study, the ability to accurately determine an unknown concentration of formaldehyde was made. Using the standard addition method once more, an aliquot of the sample of unknown concentration was made to the analytical solution, followed by a series of known formaldehyde additions. Figure 4 illustrates one plot from a series. The rest can be found in the Supporting Information. A plot was formed of current vs. concentration, where the current for the unknown addition would correspond to 'zero' concentration. To identify the unknown concentration, the line of best fit was then extrapolated back until intercept with the $x$-axis. This modulus of the $x$-intercept then gave the concentration of the unknown. The 'unknown' in Figure 4 was $0.18 \mathrm{mM}$, extrapolation of the line gave an intercept of $0.17 \mathrm{mM}$.

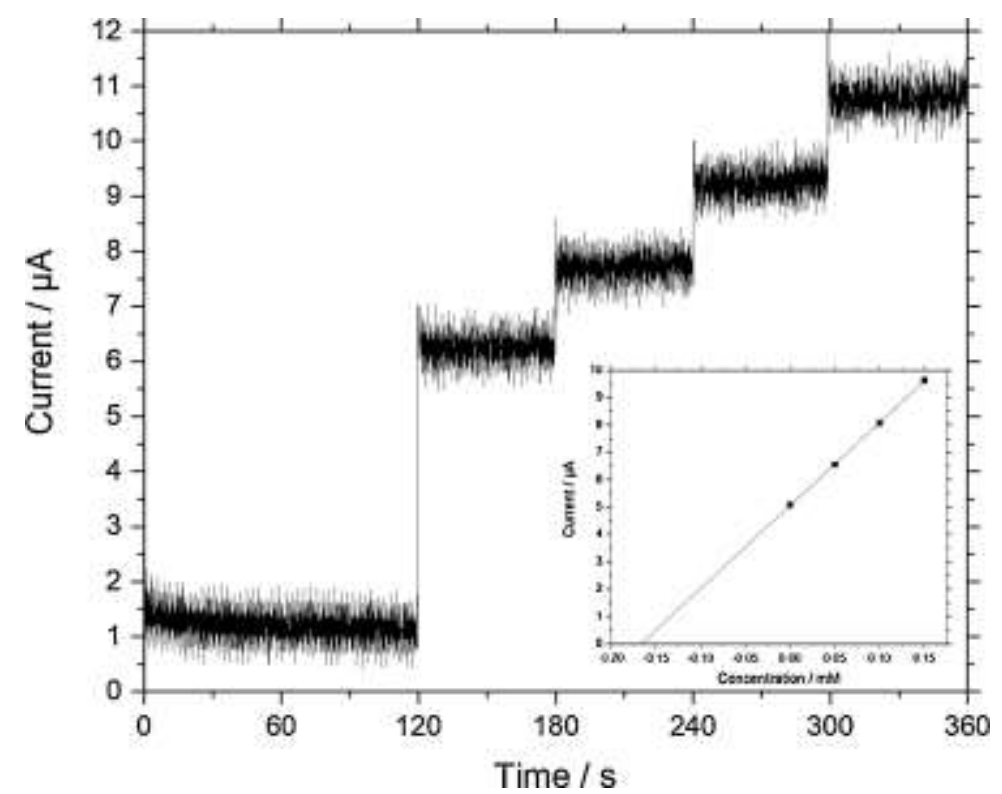

Figure 4: Standard additions of formaldehyde under chronoamperometric conditions, using the Ni-GC electrode under constant, fast stirring. Addition of unknown concentration sample of formaldehyde in Milli-Q, followed by three additions of $0.05 \mathrm{mM}$ formaldehyde in Milli-Q water. Modulus of the $x$-intercept indicates the unknown concentration.

Further 'unknown' concentrations were determined using this method to evaluate the accuracy of the technique with respect to the limit of detection of $10^{-5} \mathrm{M}$. The percentage accuracy was found to be between 0 to $12.5 \%$. It was also found that the $\mathrm{Ni}-\mathrm{GC}$ was 
durable for about 10 to 15 tests when handled with care. The sensitivity over the lifespan of the Ni-GC remained consistent, with values around $20 \mu \mathrm{A} / \mathrm{mM}$, showing excellent reproducibility.

\subsubsection{Interference tests}

Nickel modified electrodes are very sensitive to a range of small organic molecules, notably certain alcohols and glucose. It was therefore necessary to ascertain the relative sensitivity of the Ni-GC electrode to the formaldehyde whilst in the presence of potential interferants. The compounds selected as potential interferants in this study were methanol and formic acid, as they are the other solution-based compounds likely to be formed during electrochemical $\mathrm{CO}_{2}$ reduction in aqueous solutions.

In a chronoamperometric study, the potential interfering species were added in a similar standard addition fashion as with the calibration data above. Figure 5 shows the results of a series of formaldehyde additions at $0.01 \mathrm{mM}$, followed by methanol and the formic acid at $0.01 \mathrm{mM}$, and subsequently at $0.1 \mathrm{mM}$. Finally an addition of $0.1 \mathrm{mM}$ formaldehyde is made. Whilst not very discernable for methanol and formic acid, these additions were made at 50 second intervals. There is a slight, gradual rise in the potential following the addition of the methanol and formic acid, but this is only at $0.1 \mathrm{mM}$ and it is insignificant compared to the addition of formaldehyde. The Ni-GC electrode therefore demonstrates excellent selectivity towards formaldehyde compared to methanol and formate.

It is worth noting that due to the use of highly alkaline $\mathrm{KOH}$ as the analysis medium in the electrochemical studies, there is the potential for the Cannizzaro reaction to take place [36].This reaction is recognized as the spontaneous disproportionation of formaldehyde to methanol and formic acid. It is evident from the data shown in Figure 5 that this is not occurring on the time scale of the experiment, however. As formic acid and methanol are showing no response on the $\mathrm{Ni}-\mathrm{GC}$ electrode, but formaldehyde is showing a large and linear response, it may be assumed that on the timescale of the analysis that formaldehyde is not altered. 


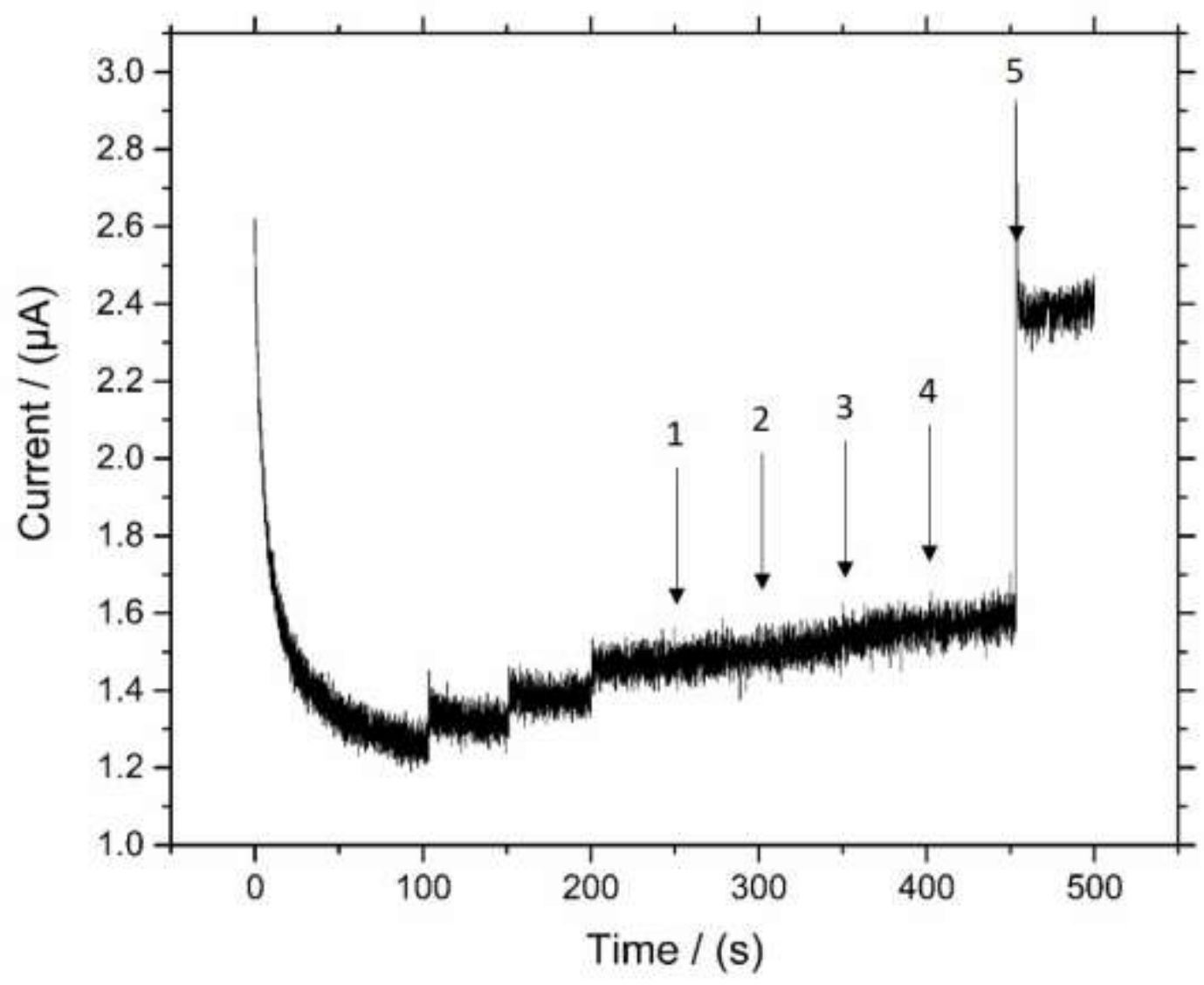

Figure 5. The catalytic response of the Ni-GC electrode in $1 \mathrm{M} \mathrm{KOH}$ to additions of $0.01 \mathrm{mM}$ formaldehyde at 100, 150 and $200 \mathrm{~s}$, followed by additions of methanol and formic acid. 1 methanol $0.01 \mathrm{mM} ; 2$ - formic acid $0.01 \mathrm{mM} ; 3$ - methanol $0.1 \mathrm{mM} ; 4$ - formic acid 0.1 $\mathrm{mM} ; 5$ - formaldehyde $0.1 \mathrm{mM}$.

\subsection{Spectrophotometric determination}

While the electrochemical approach offers the rapid analysis of a post reaction matrix for the determination of formaldehyde, it is not $100 \%$ selective, and a complimentary method of assessing formaldehyde is also required. Due to the likelihood of a complex matrix, and the extensive pre-processing necessary, the use of the standard 2,4-DNPH method of analyzing formaldehyde was rejected. Instead, a simpler, and more flexible method utilizing the Hantzsch [14] reaction was investigated. 
The UV spectrum of the derivatized formaldehyde gave a single absorption peak at ca. $412 \mathrm{~nm}$. This wavelength was used to obtain calibration plots for three sets of formaldehyde. The results of the calibration analysis are shown, overlaid in Figure 6. Very little discrepancy is observed between batches, with a near identical absorption being observed.

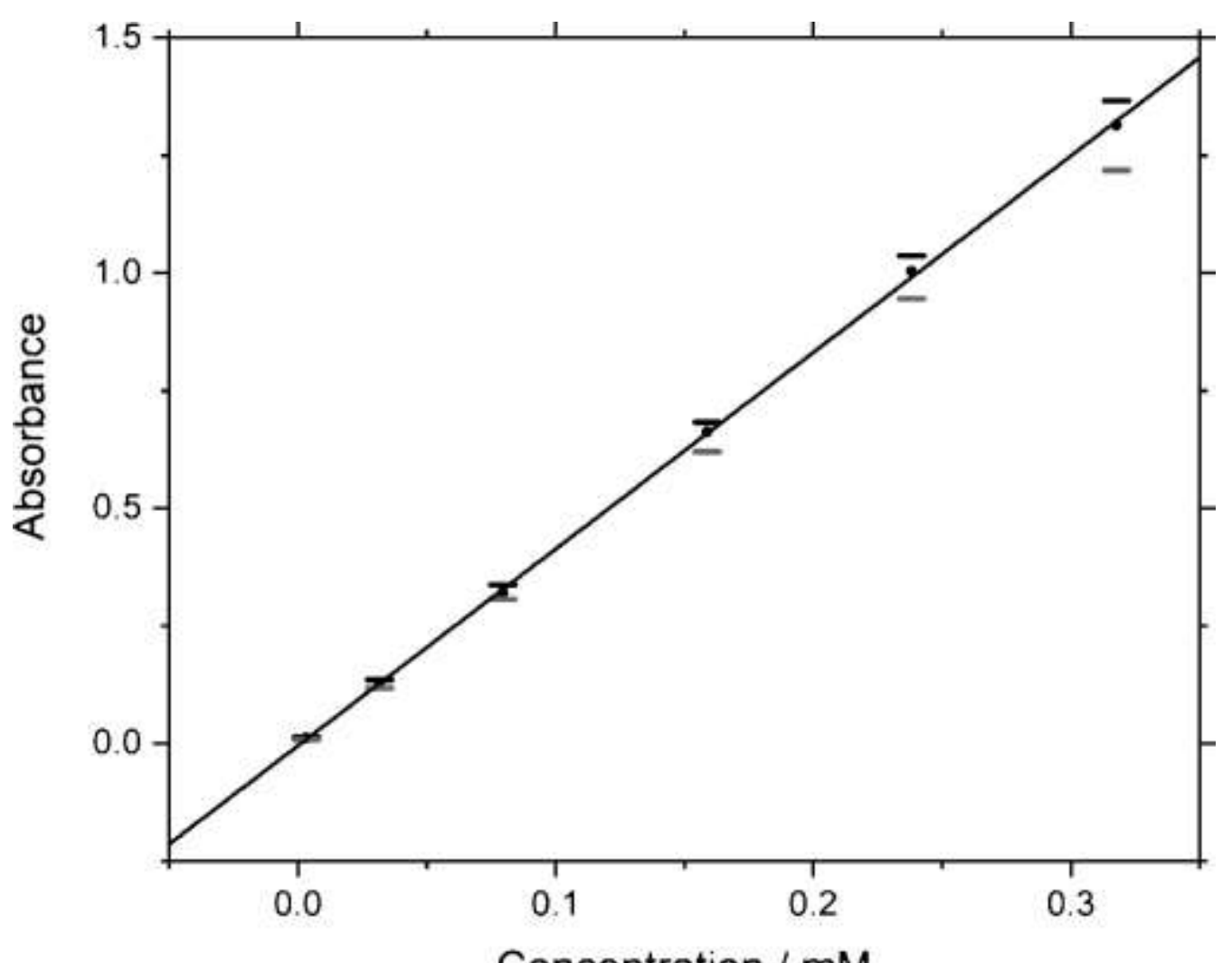

Concentration / $\mathrm{mM}$

Figure 6: UV/vis calibration data for the DDL derivatized formaldehyde where $n=3$.

The calibration via UV/vis spectrophotometry and using HPLC (results not shown) shows a highly linear response to the formaldehyde concentrations, and a wide linear range 2 orders of magnitude $(0.1-10$ ppm, $0.003-0.3 \mathrm{mM})$. Instrument precision was measured by analysing the same standard three times. The standard deviation of the mean of these three samples was $+/-2.43 \%$ for HPLC and $+/-0.00 \%$ for UV/Vis. The calculated detection limit determined for this method was $6 \times 10^{-6} \mathrm{M}$, yet the lowest observable standard was 3 $\times 10^{-6} \mathrm{M}(0.1 \mathrm{ppm})$. The calibration was tested against using three aqueous formaldehyde samples independently made up of the operator; mean accuracy was found to be $99.34 \%$. This is again, a much better accuracy than obtained using the electrochemical method. Furthermore, the Hantzsch reagent is not reactive to the potential interfering species methanol and formic acid. Consequently, these had no effect on the UV/vis spectra 
obtained. Based on the UV-Vis method, the extinction coefficient was $0.1434 \mathrm{~L} \mathrm{~mol}^{-1} \mathrm{~cm}^{-1}$ (this is the slope of the line of the calibration). HPLC data can be found in SI section

3.3. Comparison of electrochemical and spectrophotometric methods in real sample analysis

The determination of low formaldehyde concentrations in more complex matrices was then investigated by both electrochemical and spectrophotometric methods. In $0.1 \mathrm{M}$ potassium hydrogen carbonate, a matrix typical of electrochemical $\mathrm{CO}_{2}$ reduction, a volume of formaldehyde was added. Standard additions of this solution found there to be negligible difference in the determination of formaldehyde to analysis in pure Millipore water. Equally, the spectrophotometric method determined the formaldehyde concentration with zero percent error.

A subsequent experiment observed the quality of determination for analysis of naturally occurring water sources. This experiment was carried out using (filtered) water obtained from a stagnant pond. The water was first analysed as found, and no formaldehyde was detected. It was then spiked with a known concentration of formaldehyde (10 mM). The same standard addition method was carried out with this, with the first addition being an "unknown" amount of the pond water formaldehyde sample, followed by three additions of known concentration formaldehyde in Milli- ${ }^{\circledR}$ ultrapure. Figure 7 illustrates one of the plots obtained, with the corresponding calibration plot in-set. The 'unknown' in Figure 7 was $0.06 \mathrm{mM}$, extrapolation of the line gave an intercept of -0.06 . Further analysis in the pond water matrix found there to be a consistent percentage error was typically zero, though some tests gave up to $12.5 \%$ error. A summary of these results is given in Table S1 (Supplementary Information) and the chromatographic data given in the Supplementary Material. 


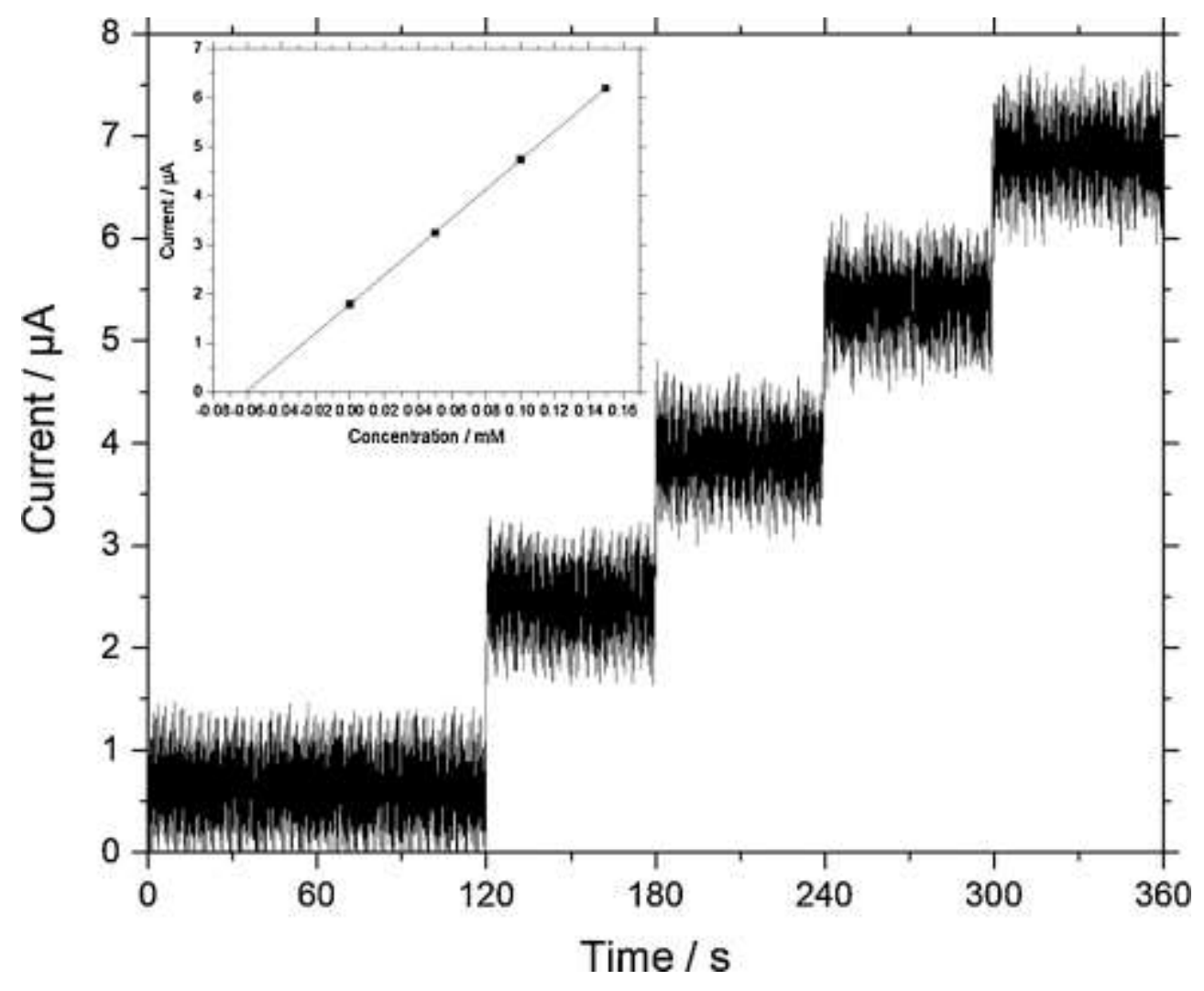

Figure 7: Standard additions of formaldehyde under chronoamperometric conditions, using the Ni-GC electrode under constant, fast stirring. Addition of unknown concentration sample of formaldehyde spiked pond water, followed by three additions of $0.05 \mathrm{mM}$ formaldehyde in Milli-Q.

Comparative analysis was made using the spectrophotometric method. As UV/vis analysis simply requires a single point measurement of absorbance and comparing this to the calibration plot obtained in Figure 6, there are no "results" to show for these experiments. A summary of these results is given in Table S1. Again, the error between the measured and expected concentrations is most often $0 \%$, with some readings of 6 or $8 \%$ difference. It is worth noting however, that the spectrophotometric method operated at an order of magnitude higher accuracy than the electrochemical method, owing to the difference in detection limit of each approach.

The pond water analysis was carried out mainly to test whether there is interference with either methods from chlorophylls or dissolved organic carbon (DOC), for example which appears yellow/brown and is often analysed for at 254, 278 and $340 \mathrm{~nm}$, or if DOC or any other compounds would affect the Hantzsch reactions. These results show that there is no evidence of any confounding water chemistry on the measurable formaldehyde 
concentration, and that the further additions of formaldehyde in Milli- $\mathrm{Q}^{\circledR}$ ultrapure water are not affected. Therefore, it can be said that this particular contaminated water does not have an effect on formaldehyde concentration. Should the pond water have been in any way compromised in its colour, however, this would not necessarily be the case for the spectrophotometric method. Furthermore, it is of note to acknowledge that another set of tests for the spectrophotometric method, in which the glassware was not suitably prepared and cleaned (acid washed), gave very high errors in the determination of formaldehyde, of up to $800 \%$ (tabulated in the $\mathrm{SI}$ ). Whereas the electrochemical method did not require extensive cleaning and preparation of equipment, and a prepared Ni-GC electrode could be used a number of times for different samples. Freshly made electrodes prepared by consistently with a $60 \mathrm{~s}$ deposition time and applied potential also showed little to no change with regards sensitivity and current response to formaldehyde samples.

\section{Conclusions}

The application of a nickel modified glass carbon electrode (Ni-GC) to the electrochemical determination of formaldehyde in aqueous solutions is presented. The detection limit was typically of the order of $10^{-5} \mathrm{~mol} \mathrm{dm}{ }^{3}$ with the lowest determination of $1.1 \times 10^{-5}$. The detection limit is comparable to other electrochemical systems for formaldehyde determination. This was highlighted by the accurate determination of "unknown" formaldehyde concentrations. While formate and methanol were also found to be slightly electroactive to the $\mathrm{NiOOH}$ catalyst species, the formaldehyde gave a much more pronounced response, even when in a 1:10 ratio with the potential interfering species.

A complementary spectrophotometric analytical approach to analyzing formaldehyde in complex media was found to give very consistent and low detection limits of the order of $10^{-6} \mathrm{M}$. The approach was highly suited the analysis of formaldehyde in a wide range of media, and the low concentrations were highly responsive to the simple derivatization method to yield highly reproducible responses. The extinction coefficient for the derivatized formaldehyde led to the sensitive and accurate determination of unknown concentrations, following a rapid confirmatory calibration process of the UV/vis spectrophotometer.

\section{Acknowledgements}

The authors would like to acknowledge Dr. Norkus for his helpful discussions during the ICEI 2016 Singapore conference. 


\section{References}

[1] R.G. Liteplo, R. Beauchamp, M.E. Meek, R. Chénier, Formaldehyde, World Health Organization, Geneva, 2002.

[2] Agency, For, Toxic, Substances, And, Disease, Et Al., Toxicological Profile For Formaldehyde, In: P.H. Service (Ed.)1999.

[3] A. Mavropoulos;, D. Newman, Wasted Health: The Tragic Case Of Dumpsites, In: I.S.W. Association (Ed.)2015.

[4] J. Hong, W. Zhang, J. Ren, R. Xu, Photocatalytic Reduction Of Co 2: A Brief Review On Product Analysis And Systematic Methods, Anal Methods, 5(2013) 1086-97.

[5] J. Tang, X. Zou, F. Hong, Product Analysis For Co2 Electroreduction, In: J. Qiao, Y. Liu, J. Zhang (Eds.), Electrochemical Reduction Of Carbon Dioxide - Fundamentals And Technologies, Crc Press

Taylor \& Francis Group2016, Pp. 293-304.

[6] S. Fregert, I. Dahlquist, B. Gruvberger, A Simple Method For The Detection Of Formaldehyde, Contact Dermatitis, 10(1984) 132-4.

[7] M. Gryllaki-Berger, C.H. Mugny, D. Perrenoud, A. Pannather, E. Frenk, A Comparative Study Of Formaldehyde Detection Using Chromotropic Acid, Acetylacetone And Hplc In Cosmetics And Household Cleaning Products, Contact Dermatitis, 26(1992) 149-54.

[8] T. Shimomura, T. Itoh, T. Sumiya, F. Mizukami, M. Ono, Electrochemical Biosensor For The Detection Of Formaldehyde Based On Enzyme Immobilization In Mesoporous Silica Materials, Sensors And Actuators B: Chemical, 135(2008) 268-75.

[9] M. Panizza, C. Bocca, G. Cerisola, Electrochemical Treatment Of Wastewater Containing Polyaromatic Organic Pollutants, Water Research, 34(2000) 2601-5. [10] C. Yu, Y. Shun-Sheng, G. Zhengjun, W. Dongmei, Study On The Treatment Of Formaldehyde Wastewater By Addition Reaction, 2010 International Conference On Challenges In Environmental Science And Computer Engineering2010, Pp. 293-6. [11] U.S. Epa, Method 8315a - Determination Of Carbonyl Compounds By High Performance Liquid Chromatography (Hplc), In: U.S.E.P. Agency (Ed.), U.S. Environmental Protection Agency, United States, 1996.

[12] Y.-L. Lin, P.-Y. Wang, L.-L. Hsieh, K.-H. Ku, Y.-T. Yeh, C.-H. Wu, Determination Of Linear Aliphatic Aldehydes In Heavy Metal Containing Waters By High-Performance Liquid Chromatography Using 2, 4-Dinitrophenylhydrazine Derivatization, J Chromatography A, 1216(2009) 6377-81. 
[13] S.S.H. Ho, J.Z. Yu, Determination Of Airborne Carbonyls: Comparison Of A Thermal Desorption/Gc Method With The Standard Dnph/Hplc Method, Environmental Science \& Technology, 3(2004) 862-70.

[14] T. Nash, The Colorimetric Estimation Of Formaldehyde By Means Of The Hantzsch Reaction, Biochemical Journal, 55(1953) 416-21.

[15] Z.-L. Zhou, T.-F. Kang, Y. Zhang, S.-Y. Cheng, Electrochemical Sensor For Formaldehyde Based On Pt-Pd Nanoparticles And A Nafion-Modified Glassy Carbon Electrode, Microchim Acta, 164(2009) 133-8.

[16] G.-P. Jin, J. Li, X. Peng, Preparation Of Platinum Nanoparticles On Polyaniline-Coat Multi-Walled Carbon Nanotubes For Adsorptive Stripping Voltammetric Determination Of Formaldehyde In Aqueous Solution, J Appl Electrochem, 39(2009) 1889-95.

[17] Y. Zhang, M. Zhang, Z. Cai, M. Chen, F. Cheng, A Novel Electrochemical Sensor For Formaldehyde Based On Palladium Nanowire Arrays Electrode In Alkaline Media, Electrochimica Acta, 68(2012) 172-7.

[18] J.P. Metters, F. Tan, C.E. Banks, Screen-Printed Palladium Electroanalytical Sensors, Journal Of Solid State Electrochemistry, 17(2013) 1553-62.

[19] Q. Yi, F. Niu, W. Yu, Pd-Modified Tio2 Electrode For Electrochemical Oxidation Of Hydrazine, Formaldehyde And Glucose, Thin Solid Films, 519(2011) 3155-61.

[20] A. Safavi, N. Maleki, F. Farjami, E. Farjami, Electrocatalytic Oxidation Of Formaldehyde On Palladium Nanoparticles Electrodeposited On Carbon Ionic Liquid Composite Electrode, J Electroanal Chem, 626(2009) 75-9.

[21] J. Wang, M. Pedrero, X. Cai, Palladium-Doped Screen-Printed Electrodes For Monitoring Formaldehyde, Analyst, 120(1995) 1969-72.

[22] M.R. Baez-Gaxiola, C. Fernandez-Sanchez, E. Mendoza, Gold Cluster Based Electrocatalytic Sensors For The Detection Of Formaldehyde, Anal Methods, 7(2015) 53842.

[23] K.E. Toghill, L. Xiao, N.R. Stradiotto, R.G. Compton, The Determination Of Methanol Using An Electrolytically Fabricated Nickel Microparticle Modified Boron Doped Diamond Electrode, Electroanalysis, 22(2010) 491-500.

[24] N.R. Stradiotto, K.E. Toghill, L. Xiao, A. Moshar, R.G. Compton, The Fabrication And Characterization Of A Nickel Nanoparticle Modified Boron Doped Diamond Electrode For Electrocatalysis Of Primary Alcohol Oxidation, Electroanalysis, 21(2009) 2627-33. 
[25] J.C. Harfield, K.E. Toghill, C. Batchelor-Mcauley, C. Downing, R.G. Compton, Nickel Nanoparticle Modified Bdd Electrode Shows An Electrocatalytic Response To Adenine And Dna In Aqueous Alkaline Media, Electroanalysis, 23(2011) 931-8.

[26] K.E. Toghill, L. Xiao, M.A. Phillips, R.G. Compton, The Non-Enzymatic Determination Of Glucose Using An Electrolytically Fabricated Nickel Microparticle Modified Boron-Doped Diamond Electrode Or Nickel Foil Electrode, Sensors And Actuators B: Chemical, 147(2010) 642-52.

[27] H. Nie, Z. Yao, X. Zhou, Z. Yang, S. Huang, Nonenzymatic Electrochemical Detection Of Glucose Using Well-Distributed Nickel Nanoparticles On Straight Multi-Walled Carbon Nanotubes, Biosensors And Bioelectronics, 30(2011) 28-34.

[28] N. Qiao, J. Zheng, Nonenzymatic Glucose Sensor Based On Glassy Carbon Electrode Modified With A Nanocomposite Composed Of Nickel Hydroxide And Graphene, Microchimica Acta, 177(2012) 103-9.

[29] M. Shamsipur, M. Najafi, M.-R.M. Hosseini, Highly Improved Electrooxidation Of Glucose At A Nickel(li) Oxide/Multi-Walled Carbon Nanotube Modified Glassy Carbon Electrode, Bioelectrochemistry, 77(2010) 120-4.

[30] F. Miao, B. Tao, Electrooxidation Of Formaldehyde Based On Nickel-Palladium Modified Ordered Mesoporous Silicon, J Nanosci Nanotechnol, 13(2013) 3104-9. [31] R. Ojani, J.B. Raoof, S.R.H. Zavvarmahalleh, Preparation Of Ni/Poly (1,5Diaminonaphthalene)-Modified Carbon Paste Electrode; Application In Electrocatalytic Oxidation Of Formaldehyde For Fuel Cells, J Solid State Electrochem, 13(2009) 1605-11. [32] Y. Yu, W. Su, M. Yuan, Y. Fu, J. Hu, Electrocatalytic Oxidation Of Formaldehyde On Nickel Ion Implanted-Modified Indium Tin Oxide Electrode, J Power Sources, 286(2015) 130-5.

[33] S.N. Azizi, S. Ghasemi, F. Amiripour, Nickel/P Nanozeolite Modified Electrode: A New Sensor For The Detection Of Formaldehyde, Sens Actuators, B, 227(2016) 1-10. [34] U.S. Epa, Determination Of Carbonyl Compounds In Drinking Water By Dinitrophenylhydrazine Derivatization And High Performance Liquid Chromatography, In: E.M.S.L.O.O.R.A. Development (Ed.), U. S. Environmental Protection Agency, Cincinnati, Ohio 45268, 1992.

[35] V. Thomsen, D. Schatzlein, D. Mercuro, Limits Of Detection In Spectroscopy, Spectroscopy, 18(2003). 
[36] R. Martin, The Mechanism Of The Cannizzaro Reaction Of Formaldehyde, Australian Journal Of Chemistry, 7(1954) 335-47. 